\title{
Resonator-Based Analysis of the Combination of Mobile Handset Antenna and Chassis
}

\author{
Pertti Vainikainen, Member, IEEE, Jani Ollikainen, Outi Kivekäs, and Ilkka Kelander
}

\begin{abstract}
In this paper, the performance of the mobile phone handset antenna-chassis combination is analyzed based on an approximate decomposition of the waves on the structure into two resonant wavemodes: the antenna-element wavemode and the chassis wavemode. A double resonator equivalent circuit model is presented and used to estimate the impedance bandwidth and the respective distribution of radiation losses with typical parameter values at 900 and $1800 \mathrm{MHz}$. It is noticed that at $900 \mathrm{MHz}$, the radiation losses by the antenna element wavemode represent typically less than $10 \%$ of the total power. Thus, the antenna element works mainly as a matching element, which couples to the low- $Q$ resonant wavemode of the chassis. At $1800 \mathrm{MHz}$, the contribution of the antenna element wavemode is larger. By enhancing the coupling and by tuning the chassis resonance, it is possible to obtain an impedance bandwidth of over $50 \%$ (6-dB return loss) at both at 900 and $1800 \mathrm{MHz}$. The results given by the equivalent circuit study are fully supported by those of three-dimensional phone-model simulations, including calculation of the $S A R$ and efficiency values. In prototyping, the 6-dB bandwidth of $5.5 \%$ was obtained at $980 \mathrm{MHz}$ with a nonradiating coupling element with a volume of $1.6 \mathrm{~cm}^{3}$ on a 120 -mm-long chassis.
\end{abstract}

Index Terms-Bandwidth, handset antennas, mobile communications, quality factor, resonators, small antennas.

\section{INTRODUCTION}

$\mathbf{I}$ N MANY application areas of antennas, the effect of complex platforms has been studied extensively [1]. For mobile handsets, one of the main areas has been to investigate the properties of different antenna elements and the interaction with the user [2]-[7]. It has also been well known that the performance, especially the bandwidth, is largely defined by the combined behavior of the antenna and the phone chassis. However, the effect of the chassis is usually not analyzed, though in many published small-antenna designs the antenna is mounted on a phone chassis and from the large bandwidths obtained it can be assumed that the effect of the chassis is significant [4], [8]-[12]. Due to lack of identifying the contribution of the whole structure on the radiation, in some cases it has seemed that the fundamental limits for the bandwidth of small antennas [13], [14] have been exceeded especially at $900 \mathrm{MHz}$. For fairly large portable radios, the effect of the dimensions of the chassis on the bandwidth was studied in [15]-[17]. Recently, the effect

Manuscript received June 22, 2000; revised July 9, 2001. This work was supported in part by the Graduate School of Electronics, Telecommunications, and Automation (GETA), by the Academy of Finland, Nokia Foundation, by the Jenny and Antti Wihuri Foundation, and by the Finnish Society of Electronics Engineers.

The authors are with Helsinki University of Technology, Institute of Digital Communications (IDC) Radio Laboratory, FIN-02015 HUT, Espoo, Finland (e-mail: Pertti.Vainikainen@hut.fi).

Digital Object Identifier 10.1109/TAP.2002.802085 has been investigated for smaller devices with chassis or circuit-board length in the range of $80-150 \mathrm{~mm}$, typical to current mobile-phone handsets [18]. It was noticed that the bandwidth for $900 \mathrm{MHz}$ patch antennas had very clear dependency on the length of the chassis. For some antenna types the maximum bandwidth was over five-times larger than the minimum, and the maximum bandwidth was obtained with chassis length of about $130 \mathrm{~mm}$. These results indicate that the total radiation bandwidth of the antenna-chassis combination is largely defined by the dipole-type radiation of the chassis currents, whose level further depends on whether the chassis is at resonance or not. It is also obvious, especially at $900 \mathrm{MHz}$, that the typically allowed handset-antenna element size of less than $5 \mathrm{~cm}^{3}$ is clearly too small to produce the required bandwidth if the current distribution would be restricted into the vicinity of the antenna element [13], [17]. Therefore, it is necessary to utilize the whole metallic structure of the handset to obtain the required bandwidth and thus there is also an obvious connection between the bandwidth, efficiency, and specific absorption rate (SAR) of mobile-phone antennas.

The purpose of this paper is to study the significance of the chassis effect with a rather simple but adequately accurate equivalent circuit model based on an approximate modal analysis of the fields and waves on the handset structure. For comparison, three-dimensional (3-D) simulations of phone models including the head of the user have been performed to validate the model and the results obtained with it [19], [20]. Also, experimental results are presented for prototypes designed based on the model. Finally, based on the results given by the equivalent circuit model, simulations, and prototyping, guidelines are given to utilize the proposed model in the future development of optimal solutions both in the sense of radiation properties (bandwidth, efficiency) and user interaction (SAR). The presented work is based on patch antenna and planar inverted-F antenna (PIFA) -type antenna elements, but the results can be generalized to apply to any antenna elements as soon as the necessary parameters, especially the radiation quality factor of the element itself, are known.

\section{EQUivalent Circuit ModeL}

\section{A. Wavemodes of the Antenna-Chassis Combination}

The maximum dimension (the length) of current mobile handsets is less than half a wavelength at $900 \mathrm{MHz}$. The other dimensions including those of the antenna elements are clearly smaller. Thus, the structure can support only a few significant wavemodes. In this paper, we divide the structure into two significant parts: the antenna element and the chassis. In current 


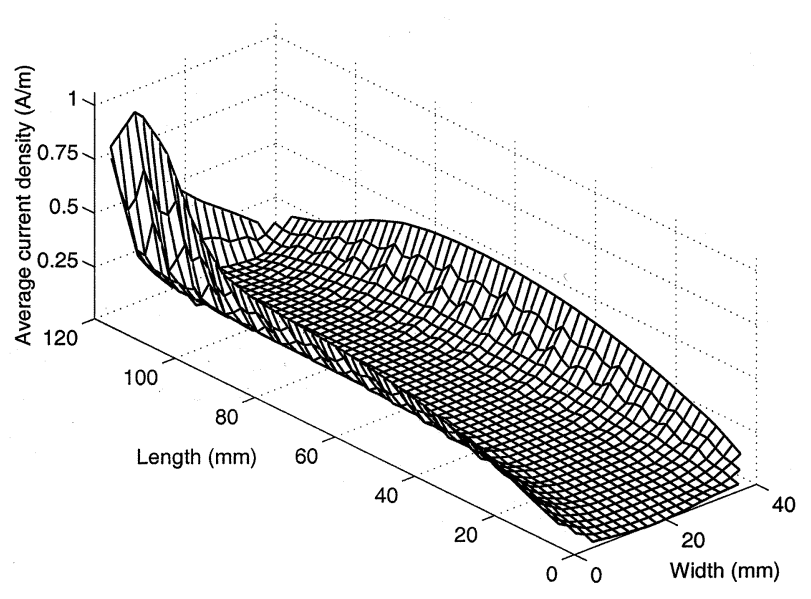

Fig. 1. Current density distribution of the combination of a patch antenna and phone chassis. The concentration of the current for the antenna-element wavemode is clearly seen, as well as the sinusoidal distribution and edge maxima for the dipole-type wavemode of the chassis.

mobile handsets the antenna element is usually self-resonant and has some characteristic wavemode, which is typically a slow-wave mode like in normal mode helices or a reactively loaded quasi-TEM mode like in patch antennas (or PIFAs). The fields and currents of this wavemode are concentrated in the vicinity of the small antenna element and for this mode the chassis acts as a groundplane with confined currents creating the mirror-image effect for the antenna element. The length of the handset chassis or circuit board is clearly larger than the width, or especially the thickness, and therefore the structure supports also single-wire or thick-dipole type current distributions. Here, the fields and currents are distributed over the whole structure and thus, the distribution is clearly less concentrated spatially than the antenna-element wavemode. An example of a typical current distribution of the antenna-chassis combination is shown in Fig. 1, where the distributions of the patch-antenna element and chassis wavemodes are clearly distinguished. Due to the small electrical size of the antenna and the chassis, the frequency response of the wavemodes is characterized by lowest order resonances including one to three standing wave "lobes." The basic idea presented here is that while considering its radiation and circuit properties, the antenna-chassis combination can be described by combining the separate radiation and impedance characteristics of the wavemodes of the antenna element and the phone chassis. These characteristics are defined by the respective current distributions and resonant responses. Furthermore, as is well known, the impedance and the surface field distribution of an antenna are connected through a surface integral relationship [21]. Thus, it is also possible to study the relationships between impedance properties and radiated fields, especially what comes to resistive components of the impedance and the radiated power. The contributions of each part of the combination to radiated power, near fields, and bandwidth are largely defined by the relative amplitudes of the wavemodes, which can be selected by tuning the coupling between the wavemodes. Accurate determination of the radiation properties of the antenna-handset combination requires, of course, a complete analysis of the combined wave distribution with, for instance,

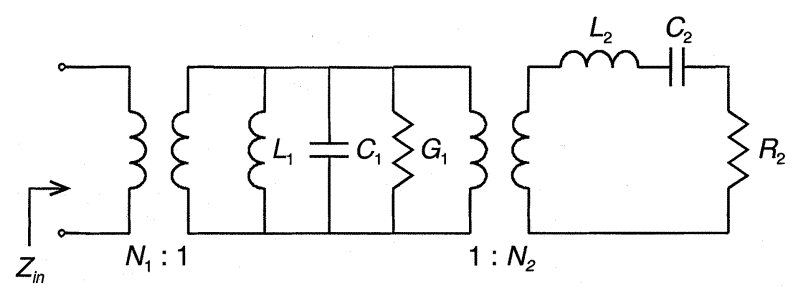

Fig. 2. Circuit model of the combination of a single-resonant antenna and chassis.

numerical methods; but the modal analysis presented here can be used to identify main aspects of the radiation, especially in cases where either one of the wavemodes dominates clearly. As the impedance bandwidth, which is also defined by the coupling and the radiation quality factors of the wavemodes, is often the most critical design parameter of a handset antenna; the coupling between the modes and thus also many properties of the radiation are largely defined by the bandwidth requirement as seen below.

\section{B. Equivalent Circuit}

When investigating a small antenna element located on a phone chassis, it can be noticed that the currents flowing on the chassis are induced there through electromagnetic coupling from the antenna element, which is further excited by its feed. As the antenna is self-resonant and the length of the chassis is often close to some multiple of half wavelength, the obvious lumped-component equivalent circuit model of the combination is that of coupled parallel and series resonators as shown in Fig. 2. In Fig. 2, resonator 1 represents the antenna element and resonator 2 the phone chassis. While studying the performance of the circuit, the type of the resonators is insignificant except that there must be both a parallel and a series resonator as normally in the case of electromagnetically coupled resonators [22]. However, it is known that the impedance behavior of a shorted probe-fed patch antenna (or PIFA) is close to that of a parallel resonant circuit and thus in this paper the circuit in Fig. 2 was selected. For a PIFA also, the inductance of the probe can be included in the model but it is not significant from the main results point of view. Furthermore, resonator 1 can also be considered to be a nonradiating matching circuit providing only coupling to the radiating chassis resonance. The input impedance $Z_{i n}$ of the circuit model in Fig. 2 is

$$
\begin{aligned}
Z_{i n} & =\frac{N_{1}^{2}}{\frac{1}{\mathrm{j} \omega L_{1}}+\mathrm{j} \omega C_{1}+G_{1}+\frac{N_{2}^{2}}{\mathrm{j} \omega L_{2}+\frac{1}{\mathrm{j} \omega C_{2}}+R_{2}}} \\
& =\frac{N_{1}^{2}}{G_{1}\left[1+\mathrm{j} Q_{a}\left(\frac{\omega}{\omega_{r a}}-\frac{\omega_{r a}}{\omega}\right)\right]+\frac{N_{2}^{2}}{R_{2}\left[1+\mathrm{j} Q_{c}\left(\frac{\omega}{\omega_{r c}}-\frac{\omega_{r c}}{\omega}\right)\right]}} .
\end{aligned}
$$

\section{Parameters of the Resonators}

In (1), the chassis resonant frequency is $f_{r c}=\omega_{r c} / 2 \pi=$ $1 /\left(2 \pi \sqrt{L_{2} C_{2}}\right)$ defined by the length $l$ of the chassis as

$$
f_{r c}=\frac{1}{2} \frac{n c}{l+2 \Delta l}=\frac{n c}{2 l}-\Delta f .
$$


Here, $n$ is the multiple number of half wavelengths for the resonant mode (order of the resonance) $c=3 \cdot 10^{8} \mathrm{~m} / \mathrm{s}$ and $\Delta l$ is the open-end extension of electrical length of the chassis causing the shift of $\Delta f$ of $f_{r c}$ from the nominal value. It must be noticed here that the antenna element may also have an effect on the resonant frequency of the chassis wavemode as some part of the currents of this wavemode may flow in the antenna element. Typical examples are monopole-type antennas, which are usually located at the end of the chassis and thus increase the electrical length of the chassis as well.

The unloaded quality factor $Q_{c}=1 /\left(\omega_{r a} R_{2} C_{2}\right)$ of the chassis represents all losses of the chassis wavemode. In addition to the radiation, these also include conductive losses, which should be minor and dielectric losses from possible lossy dielectrics in the phone structure and especially those from the user's body. The contribution of each loss mechanism can be described with the respective quality factor and the total $Q_{c}$ is obtained in the standard manner as the inverse of the sum of inverses of individual quality factors. In the circuit model, the different quality factors are described by splitting $R_{2}$ into several loss resistors in series. The conductive and dielectric quality factors can be found by using the well-known analysis methods for resonant cavities [23]. The radiation quality factor depends on the transversal dimensions so that for larger equivalent radius of the dipole-type chassis structure the quality factor is lower [21]. By simulations, it was found out that for a thin $(t \approx 0)$ metal plate with length $l=80 \cdots 130 \mathrm{~mm}$ and width $w=40 \mathrm{~mm}$, the first-order $(\lambda / 2)$ resonance at the $1-\mathrm{GHz}$ range has $f_{r c} \approx(0.73 \cdots 0.78) \cdot c / 2 l$ and the unloaded quality factor $Q_{c} \approx 2.3 \cdots 2.8$. The respective values for the second-order resonance close to $2 \mathrm{GHz}$ were found to be $f_{r c} \approx(0.83 \cdots 0.85) \cdot c / l$ and $Q_{c} \approx 4$. According to the simulations, the increase of the chassis thickness to $t=3 \mathrm{~mm}$, which is typical for modern handsets, has only minor effects on $f_{r c}$ and $Q_{c}$. From these values it can be seen that the lumped-element single-resonator model of the chassis is somewhat inaccurate, due to the overlapping of the low- $Q$ resonances; better accuracy would be obtained with a lumped dual-resonant or lossy transmission line model. However, over a relative bandwidth of about $\pm 25 \%$ around the chassis resonances, the response is fairly close to that of the single resonator circuit model and thus the main features of $Z_{\text {in }}$ can be assumed to be correct.

The unloaded quality factor $Q_{a}=\omega_{r a} C_{1} / G_{1}$ of the antenna element alone represents again all losses of the respective wavemode: radiation, conductive, and dielectric losses and similar approach as in the case of the chassis wavemode can be used. For high- $Q$ antenna elements the internal dielectric and conductive losses may be significant. The separate radiation quality factor of the antenna element is somewhat difficult to determine as the chassis effect is so significant in all typical implementations. Based on the fact that the radiation of an antenna is defined by its current distribution it can be claimed that the radiation quality factor of the wavemode of the planar antenna alone can be obtained by investigating the respective antenna situated on a large ground plane [17]. The basis for this estimation is that the current distribution inside and close to the planar antenna does not change very much when the antenna is located on a phone chassis. Another way to get insight to this question is to study the bandwidth of the antenna-chassis combination as a function of the length of the chassis as done later in this paper. The chassis lengths at which the bandwidth has its minimums can be assumed to represent the cases, where the contribution of the chassis radiation is small and the bandwidth obtained is that given by the antenna element alone. It is also useful to study the ultimate limit for the radiation quality factor of a small antenna [13], [14], because it is typical for many antenna types like PIFAs that their radiation quality factor is approximately inversely proportional to volume and thus, it has almost constant ratio to the smallest possible radiation quality factor. For a typical mobile handset, the maximum value for the radius of the sphere enclosing the antenna element and thus, also the major parts of its wavemode, is around $15 \mathrm{~mm}$. This gives the minimum radiation quality factor of around 50 at $900 \mathrm{MHz}$, and around 7 at $1800 \mathrm{MHz}$ for a lossless single-mode antenna. The quality factor of practical handset antenna elements is several times higher than the theoretical limit [13], [17]. Based on all this information it can be assumed that the practical minimum values are $Q_{a} \approx 100$ for $900 \mathrm{MHz}$ and $Q_{a} \approx 15$ for $1800 \mathrm{MHz}$. For the case where the first resonator is a nonradiating matching circuit, the quality factor is in the ideal case infinite $\left(G_{1}=0\right)$ and also in practice values on the order of $Q_{a} \approx 500$ can be obtained below $2 \mathrm{GHz}$. The resonant frequency $f_{r a}=\omega_{r a} / 2 \pi$ of the antenna element can be tuned rather simply to the required frequency of operation (often, however, at the expense of $Q_{a}$ ) by using methods like dielectric or reactive loading or meandering [8], [10], [12], [18], and [24]. The ideal case would be $f_{r c}=f_{r a}$, but this is more and more difficult to achieve at $900 \mathrm{MHz}$ as the phones get smaller and the typical chassis length is clearly less than $120-130 \mathrm{~mm}$, which would give $f_{r c} \approx 900 \mathrm{MHz}$.

\section{Coupling Factors}

The coupling factor $N_{2}$ in (1), giving the impedance scaling factor between the resonators is defined by the coupling of the antenna to the chassis resonance. For an antenna located near the end of the chassis, the coupling takes place mostly through electric fields and is thus capacitive. The coupling can be tuned by changing the mutual capacitance between the antenna and chassis wavemodes. This in turn is affected by the height and width of the antenna and its location versus the electric fields of the chassis wavemode. Respectively, magnetic coupling can be arranged with loop-type structures located close to the magnetic field maximum of the chassis wavemode. The coupling factor $N_{1}$ of the dual-resonant circuit to the feed line can usually be tuned over a broad range of values, e.g., by changing the feed location of the antenna.

\section{TheORETical Results With the EQUIVALENT CIRCUIT MODEL}

As mentioned above, there is a connection between the radiation bandwidth of the antenna-chassis combination and the relative amplitudes of the modes. It was also shown that the radiation quality factor of the antenna element is much higher than 


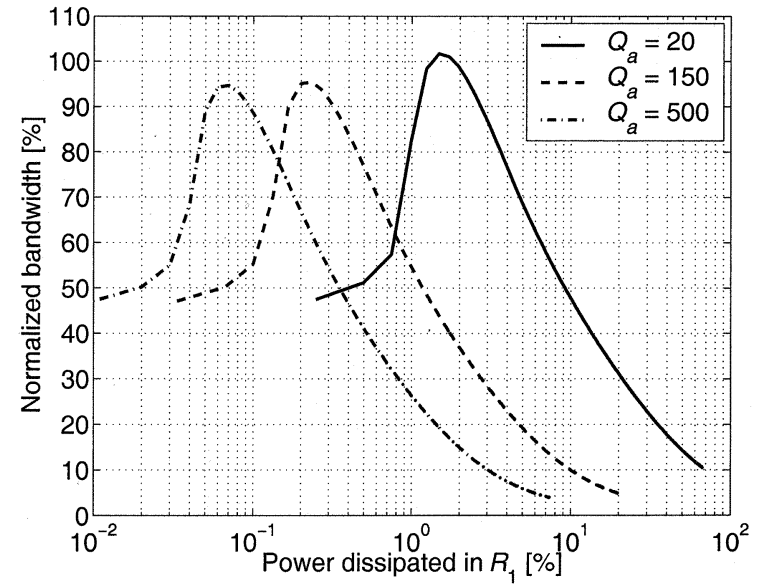

Fig. 3. Bandwidth as a function of power dissipated in the antenna element for different values of $Q_{a}$.

that of the chassis. This being the case, it was interesting to use the dual-resonant equivalent circuit to study the following items:

- The maximum bandwidth obtained as a function of coupling $\left(N_{2}\right)$ between the two resonators. The input coupling factor $N_{1}$ was tuned to obtain maximum bandwidth for each value of $\mathrm{N}_{2}$.

- The distribution of power between the resonators for different values of bandwidth to estimate the contributions of the wavemodes on the radiated power (if no other losses are present) or, correspondingly, the power lost in the nonradiating matching circuit.

- The effect of the antenna quality factor $Q_{a}$ on the bandwidth and distribution of power. As shown above, the chassis quality factor is clearly lower, and fairly constant, and thus, an average value of $Q_{c}=3$ was used.

- The effect of the difference between the resonant frequencies of the chassis and the antenna to investigate, especially the situation caused by a "too short" handset.

In the calculations, the matching criterion was $L_{\text {retn }} \geq 6 \mathrm{~dB}$, which is rather typical for small internal antennas [18]. Results for other matching criteria can be obtained with respective scaling factors. For example, for single-resonant antennas the optimal 6-dB bandwidth is about $130 \% / Q_{a}$ and for $10-\mathrm{dB}$ bandwidth, about $70 \% / Q_{a}$ [17]. Thus, the scaling factor to get the 10-dB bandwidth from the $6-\mathrm{dB}$ bandwidth is about 0.5 . All frequencies and bandwidths have been normalized by defining $f_{r a}=1$ everywhere. In Fig. 3, the results for the normalized bandwidth (compared to $f_{r a}=1$ ) as a function of the power $P_{d 1}$ dissipated (lost or radiated) in resonator 1 at $f=1$ are shown for $Q_{a}=20,150$ or 500, respectively, for the best possible case when $f_{r c}=f_{r a}$. The basis for such values for $Q_{a}$ was described generally in Section II. The connections of the selected values to practical implementations are as follows:

- $Q_{a}=20$ is the typical value obtained for a 4-mm-thick air-filled single-resonant quarterwave patch (or PIFA) at around $2 \mathrm{GHz}$ on a large ground plane [17]. However, at $900 \mathrm{MHz}$, this value is not possible to achieve with a patch, PIFA, or helix, fulfilling the size requirement for a handset-antenna element.

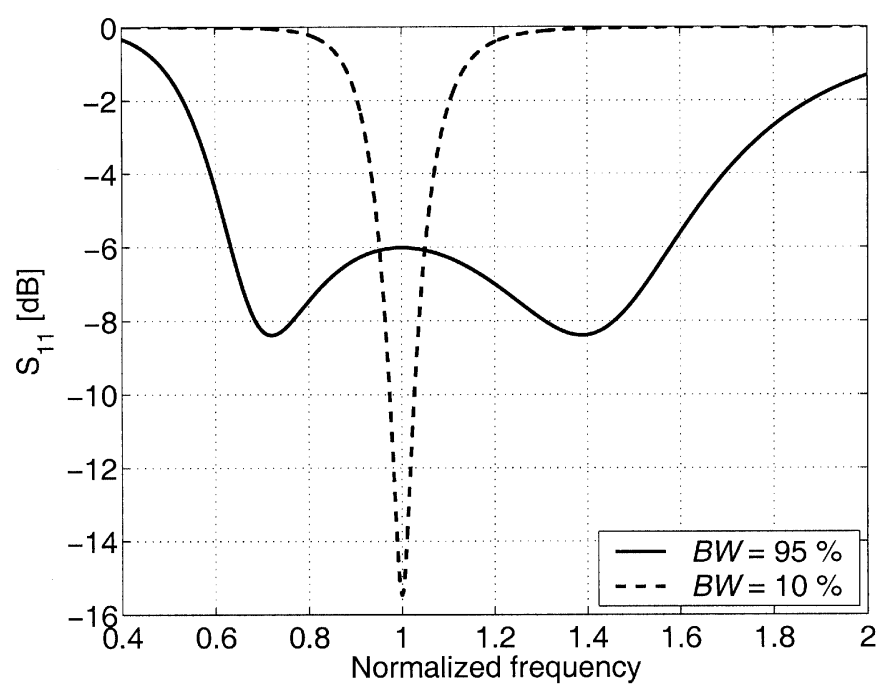

Fig. 4. Examples of frequency responses of the reflection coefficient with $Q_{a}=500$ for the maximum bandwidth of Fig. 3 (solid line) and for $10 \%$ bandwidth (dashed line).

- $Q_{a}=150$ is the estimated radiation quality factor if the 4-mm-thick antenna element mentioned above is tuned to resonance at $900 \mathrm{MHz}$, but the volume is not changed (to meet the volume requirement). The estimation is based on the inverse proportionality of radiation quality factor to the volume of a small antenna in wavelengths [13], [14] and supported by results of simulations with different lengths of the ground plane (see Section IV-A). This value can also be used for a nonradiating reactive coupling element with matching circuit consisting of high-quality lumped elements.

- $Q_{a}=500$ can be estimated to be a typical quality factor obtained with a reactive coupling element with distributed microstrip matching circuit on high-quality substrate or with a small self-resonant antenna/coupling element [12].

In Fig. 3, the coupling to resonator 2 decreases ( $N_{2}$ decreases) as $P_{d 1}$ increases. When $P_{d 1}=0 \%$, all power is radiated by the chassis, we have the single-resonant case and the 6-dB bandwidth obtained is about $130 \% / Q_{c} \approx 45 \%$. With very loose coupling to the chassis resonance (beyond the right end of the scale in Fig. 3) we get $P_{d 1}=100 \%$, all power is radiated by the antenna-element wavemode, and again we have a single-resonant case now with $6-\mathrm{dB}$ bandwidth of about $130 \% / Q_{a} \approx 7$, 0.9 , or $0.3 \%$, respectively. The maximum bandwidth seen in Fig. 3 is obtained by optimal coupling for best dual-resonant response, which typically gives somewhat more than double the bandwidth compared to the single-resonant case. If the quality factors of the two resonators are clearly different, the maximum bandwidth does not depend much on the higher quality factor as seen from Fig. 3. This indicates that huge bandwidths are possible with optimal coupling also by using practically nonradiating coupling elements.

Examples of frequency responses with different coupling levels to the chassis resonance are shown in Fig. 4 for $Q_{a}=500$. In the case of maximum bandwidth (solid line), the response is clearly dual resonant. For the typical bandwidth of about $10 \%$ (dashed line), the response looks single resonant, 


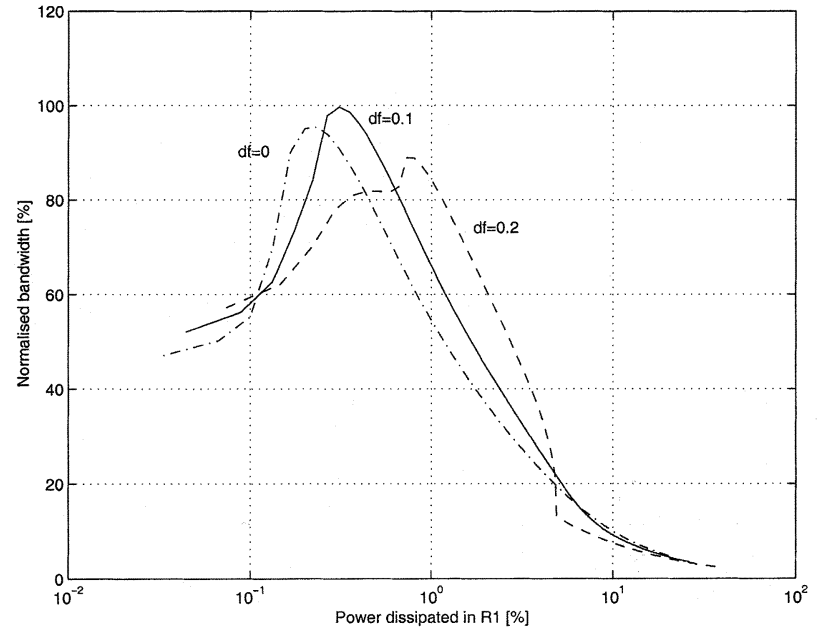

(a)

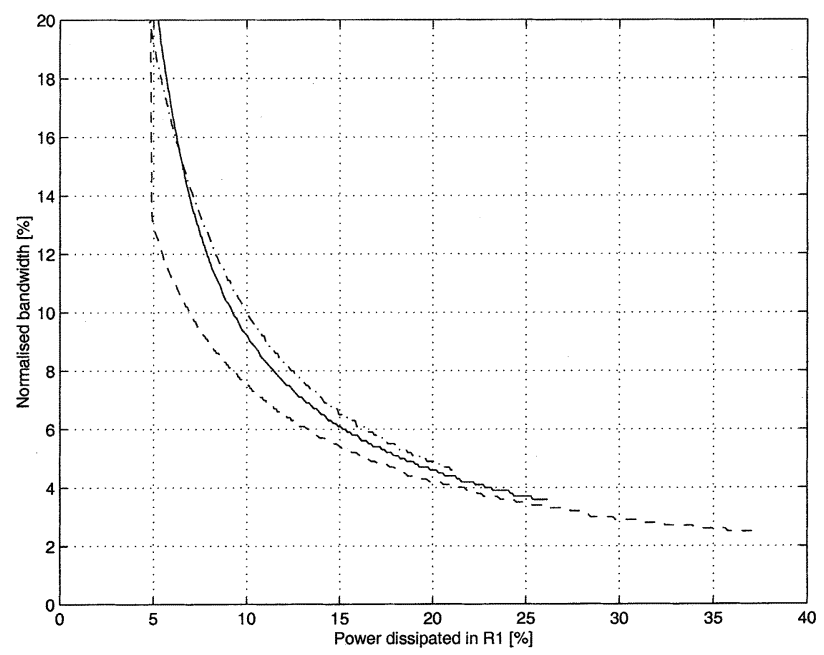

(b)

Fig. 5. (a) Bandwidth as a function of power dissipated in the antenna element for $Q_{a}=150$ and $d f=0,0.1$, and 0.2 . (b) Close-up view for small bandwidths.

though the effect of resonator 2 (chassis wavemode) is significant as seen from Fig. 3. The reason for this result is that the total bandwidth is clearly narrower than that of resonator 2. Therefore, the admittance seen from the resonator 1 toward the resonator 2 is almost constant over the whole significant bandwidth. In this case, the admittance of resonator 2 can be described with a narrow-band approximation of the equivalent circuit. This consists of a conductance $G_{2 n}$ and susceptance $B_{2 n}$ in parallel and having values, which resonator 2 represents at the center frequency of the whole circuit. The values of the components depend on the coupling factor $N_{2}$, and the difference between the center frequencies of the resonators. It is obvious that $G_{2 n}$ has maximum at $f_{r c}$ and $B_{2 n}$ is positive below $f_{r c}$ and negative above it. From the whole circuit point of view, $G_{2 n}$ represents a decrease of the total effective quality factor $Q_{\text {eff }}=\omega_{\text {reff }} C_{1} /\left(G_{1}+G_{2 n}\right)$ and can also be considered as the effective chassis quality factor $Q_{\text {effc }}=\omega_{\text {reff }} C_{1} / G_{2 n}$ (not the same as $Q_{c}$ ). Now the total quality factor is obtained as $1 / Q_{\text {eff }}=1 / Q_{a}+1 / Q_{\text {effc }}$. To obtain the 6-dB bandwidth of $10 \%$ requires $Q_{\text {eff }}=13$. Because at $900 \mathrm{MHz}, Q_{a}$ is typically at least 150 , it is obvious that the effect of the coupling to the chassis wavemode is significant. The narrow-band model gives also the power distribution very simply as

$$
\frac{P_{a}}{P_{\mathrm{tot}}}=\frac{1 / Q_{a}}{1 / Q_{a}+1 / Q_{\mathrm{effc}}}=\frac{Q_{\mathrm{effc}}}{Q_{\mathrm{effc}}+Q_{a}}=\frac{Q_{\mathrm{eff}}}{Q_{a}} .
$$

Here, $P_{a}$ is the power consumed by radiation and losses in resonator 1 (antenna-element wavemode) and $P_{\text {tot }}$ the total input power to the circuit. Thus, the narrow-band approximation can be used to replace the complicated calculations with the whole circuit of Fig. 2 for the cases where $Q_{\text {eff }} \gg Q_{c}$. The main effect of susceptance $B_{2 n}$ is that it shifts the effective resonant frequency $f_{\text {reff }}$ of the whole circuit by $\Delta f_{\text {reff }} \approx-B_{2 n} /\left(2 Q_{\text {eff }}\left(G_{1}+G_{2 n}\right)\right) \approx-B_{2 n} /\left(2 Q_{\text {eff }} G_{2 n}\right)$. This shift is fairly small, because $\left|B_{2 n}\right|<G_{2 n}$ inside the 3-dB bandwidth of resonator 2, and $Q_{\text {eff }}$ is typically over 10 .

In Fig. 5, the respective results as in Fig. 3 are given for $Q_{a}=150$, when $f_{r c}=f_{r a}+\mathrm{d} f$ with $\mathrm{d} f=0.1$ or 0.2 . This describes the typical situation at $900 \mathrm{MHz}$ where the chassis is too short to be in resonance at $f_{r a}$. It can be noticed that for $\mathrm{d} f=0.1$ the effect is small on the bandwidth or power distribution. When $\mathrm{d} f=0.2$, the maximum bandwidth is still high but more problems can be expected in achieving the required performance. Actually, tighter coupling is required to obtain the same bandwidth because $G_{2 n}$ decreases as $|\mathrm{d} f|$ increases, which in practice means a larger coupling element. The obvious result obtained but not seen from Fig. 5, is that the center frequency of the band is close to $f_{r c}$ for tight coupling (large bandwidth) and close to $f_{r a}$ for loose coupling (small bandwidth).

As mentioned previously, at $900 \mathrm{MHz}$ the typical bandwidth ( $L_{\text {retn }} \geq 6 \mathrm{~dB}$ ) for a phone with an internal antenna is about $10 \%$. Thus, the results in Fig. 3 and given also by (3), indicate that the power radiated by the wavemode of the typical internal antenna element $\left(Q_{a}=150\right)$ is less than $10 \%$ of the total radiated power. In this case, it can be expected that the antenna element has only a minor effect also on properties like the body loss or $S A R$ of the phone. For $Q_{a}=20$, representing the situation at around $2 \mathrm{GHz}$, the contribution of the antenna element wavemode is clearly more significant, i.e., $P_{d 1} \approx 50 \%$ for $10 \%$ bandwidth.

\section{Simulation Results AND COMPARISON With THEORY}

\section{A. Impedance of Phone Models in Free Space}

The validity of the circuit model was studied with 3-D simulations of phone models in free space by using method of moments (MoM)-based commercial software. Simulated impedance results obtained with the software have been noticed to agree very well with experimental ones also for complicated multifrequency antenna-chassis structures [24]. Simulated impedance results for different antenna-chassis combinations are shown in Figs. 6-8. The items studied were:

- effect of increasing the coupling between the antenna and the chassis;

- effect of the difference between the resonant frequencies of the antenna and chassis;

- effect of the ground-plane length on the impedance bandwidth. 


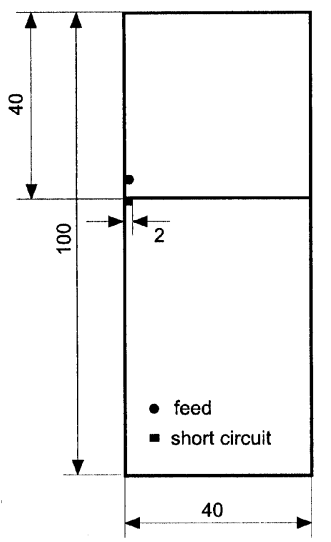

(1)
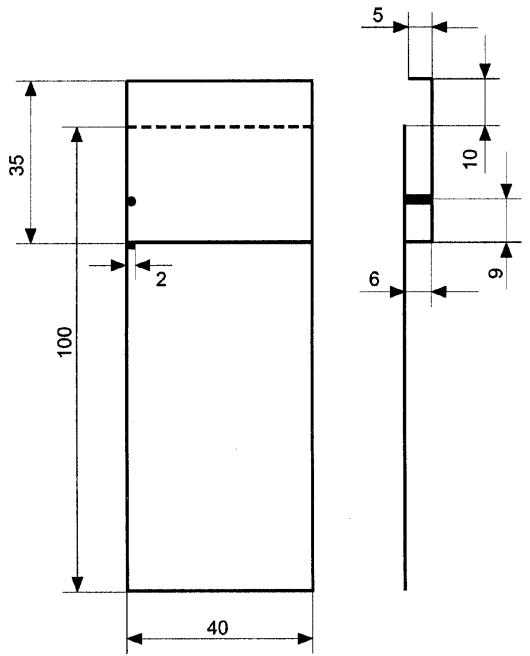

(3)
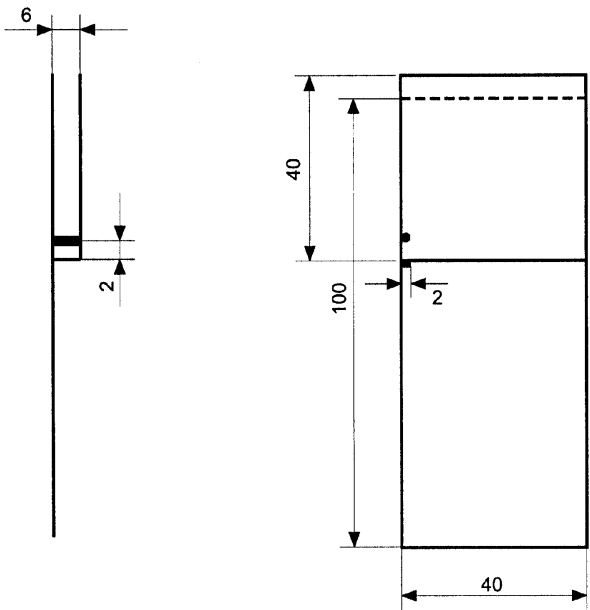

(2)

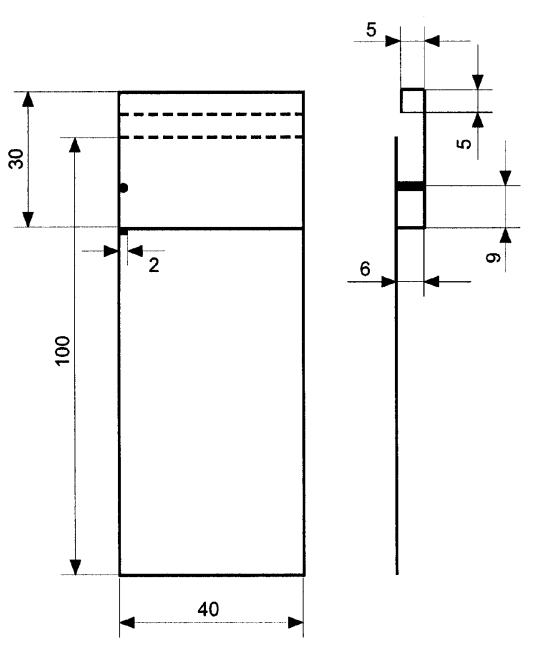

(4)

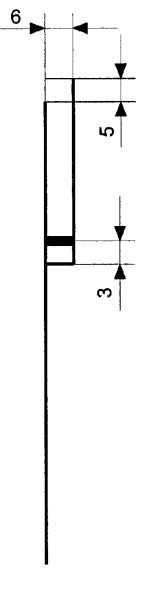

(a)
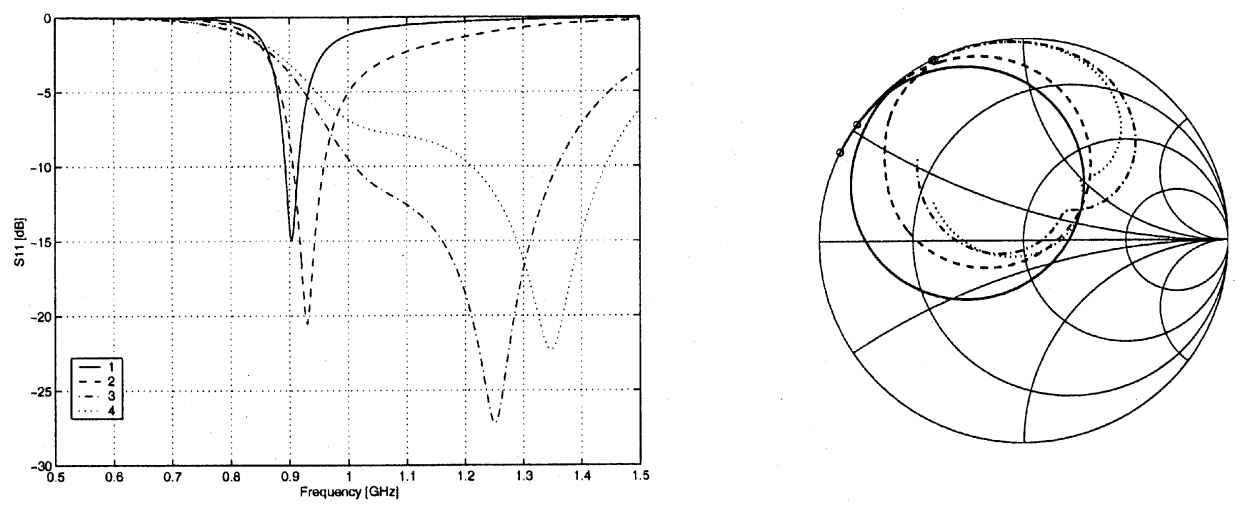

(b)

Fig. 6. (a) Shorted patch antennas for $900 \mathrm{MHz}$ with increased coupling between antenna and ground plane. Coupling increases with the ordinal number (1-4). (b) Simulated reflection coefficients for antenna structures $1-4$ of (a).

The effect of increasing the coupling between the antenna and chassis at $900 \mathrm{MHz}$ is presented in Fig. 6. The method for increasing the coupling was to extend the patch over the end of the ground plane and bend it to create strong coupling to the elec-tric-field lines at the "open" end of the chassis [see Fig. 6(a)]. The results in Fig. 6(b) show that by increasing the coupling between the driven antenna and the ground plane, the obtained bandwidth can be enhanced significantly (almost by a factor of 10). Although, a clear dual resonance can be observed in cases 3 and 4, the couplings obtained so far are not optimal. By optimizing the coupling, even greater bandwidths can be obtained as was shown in 

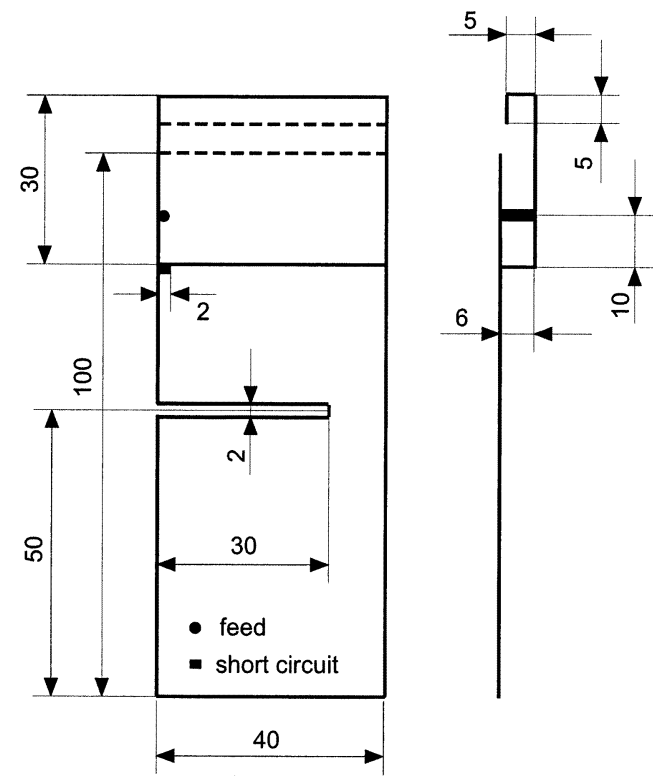

(a)
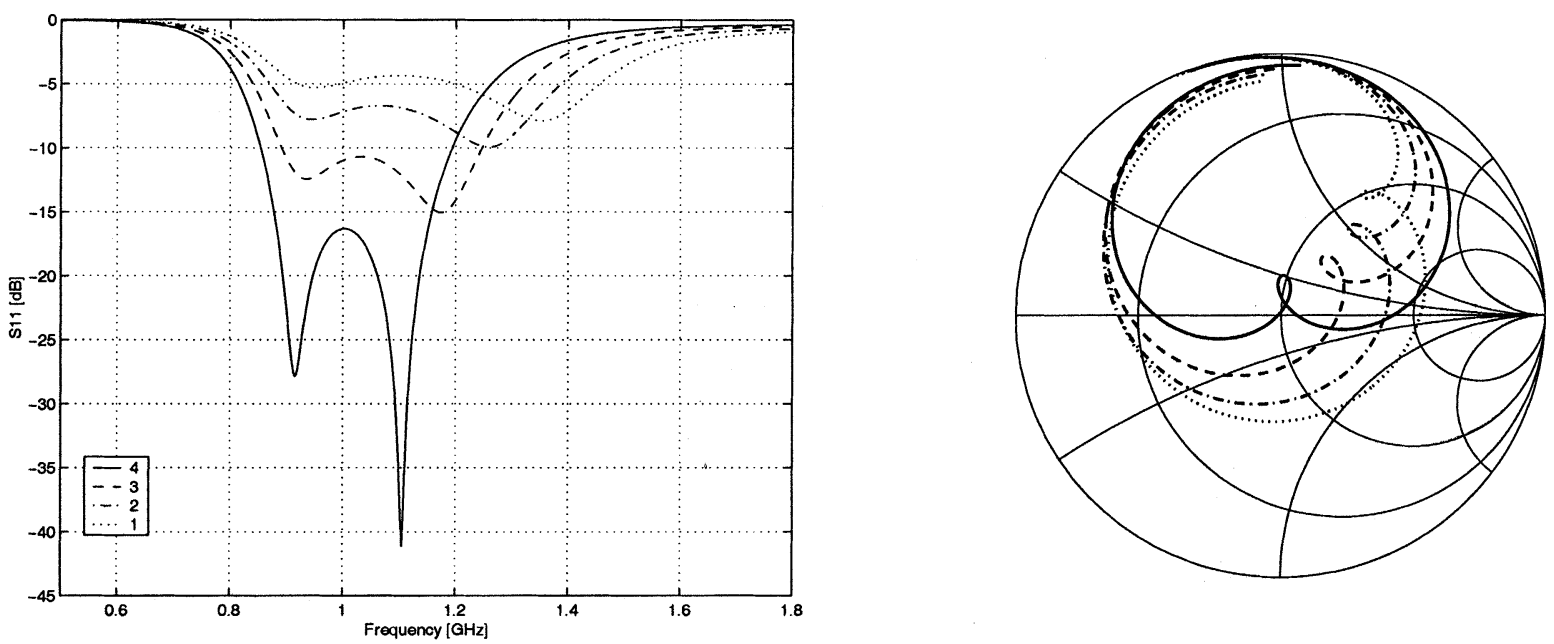

(b)

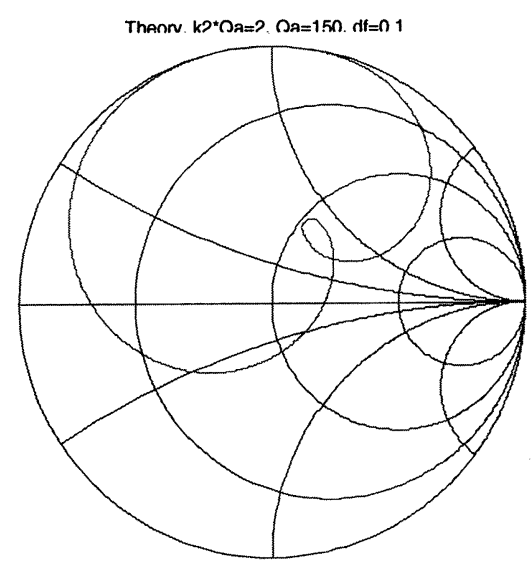

(c)

Fig. 7. (a) Shorted patch antenna with relatively strong coupling for $900 \mathrm{MHz}$ (patch length $l_{p}=30 \mathrm{~mm}$ ). Slot in the middle of the ground plane is used to decrease its resonant frequency. (b) Simulated reflection coefficients for the antenna of (a) with different patch lengths. Other dimensions as given in (a). 1) $l_{p}=$ $30 \mathrm{~mm}$; 2) $l_{p}=35 \mathrm{~mm}$; 3) $l_{p}=40 \mathrm{~mm}$;) $l_{p}=45 \mathrm{~mm}$. (c) Result obtained with the equivalent circuit of Fig. 2 and having close agreement with the case $l_{p}=40 \mathrm{~mm}$ in (b). 

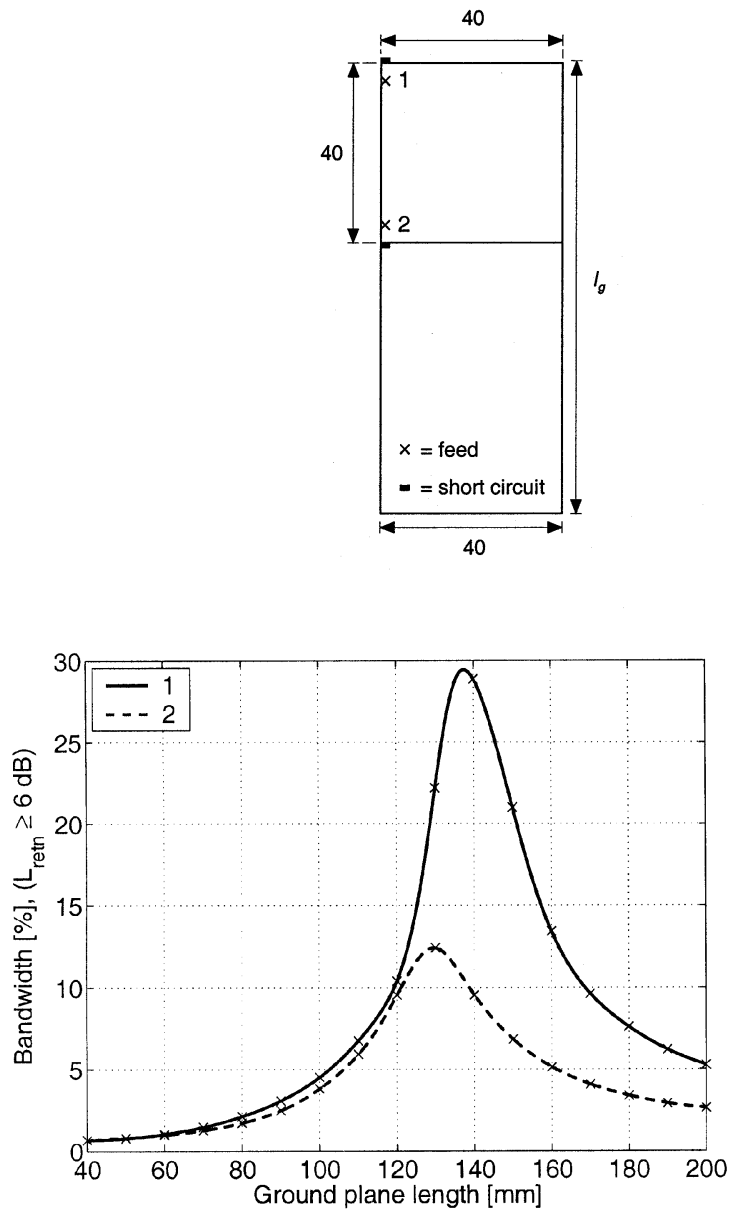

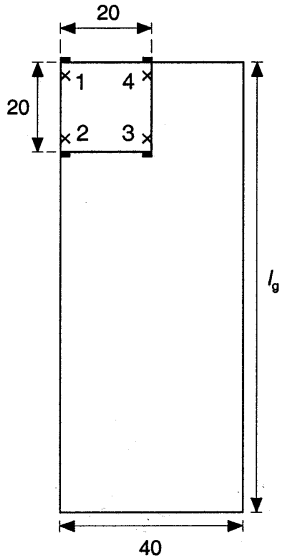

(a)

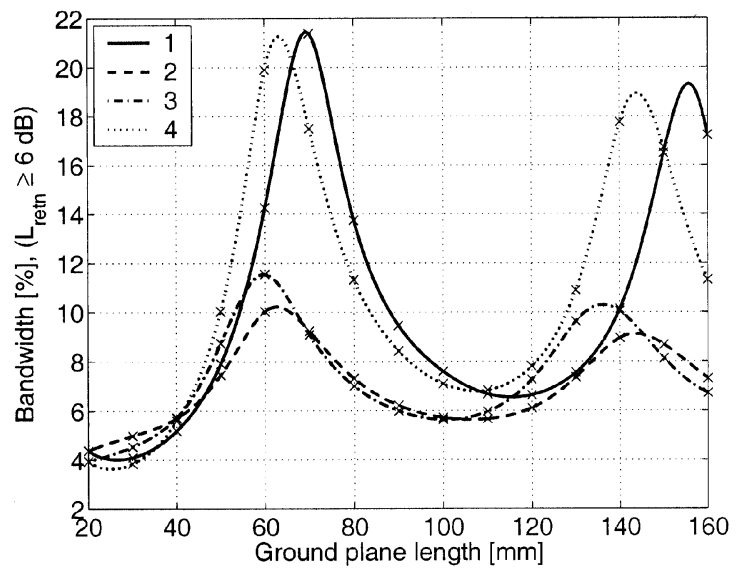

(b)

Fig. 8. (a) Antenna models for $900 \mathrm{MHz}$ (left) and $1800 \mathrm{MHz}$ (right) used in the study of the effects of ground plane length and feed position on the bandwidth of a patch antenna. The substrate thickness of both antennas was $h=5 \mathrm{~mm}\left(\varepsilon_{r}=1\right)$. (b) Simulated bandwidth $\left(L_{\text {retn }} \geq 6 \mathrm{~dB}\right)$ as a function of ground plane length for different locations and orientations of the antennas in (a). Left: $900 \mathrm{MHz}$, two different configurations; right: $1800 \mathrm{MHz}$, four different configurations.

the previous section. A similar trend could also be observed at $1800 \mathrm{MHz}$.

The effect of the difference between the resonant frequencies of the antenna and chassis is shown in Fig. 7. Here, the resonant frequency of the chassis was decreased to about $1 \mathrm{GHz}$ with a slot at the current maximum. The resonant frequency of the antenna was tuned by changing its length until the resonant frequencies of the antenna and chassis were nearly equal. As the resonant frequencies come closer to each other, the bandwidth clearly increases, as can be expected from the theoretical results. Comparing the results of Fig. 7(b) with general dual-resonant studies [22], one can estimate, that $\mathrm{d} f \approx 8 \%$ for $l_{p}=40 \mathrm{~mm}$. For this case $B W \approx 50 \%$. By taking the respective case with $\mathrm{d} f=0.1$ and $B W \approx 50 \%$ from the theoretical results in Fig. 5 and adding the effect of a short section of transmission line describing the feed arrangement of the antenna element in Fig. 7(a) one obtains very good agreement between theory and simulated result [see Fig. 7(c)]. Thus, it can be estimated that with an antenna structure such as in Fig. 7(a) one can get fairly close to the optimal coupling. However, cutting a slot into the chassis is usually not a very practical way to reduce its resonant frequency. Other means can be developed by applying to the mechanical structure of mobile handsets the well-known methods of reducing the length of dipole antennas.

The effect of the ground plane length on the impedance bandwidth of a shorted patch antenna was studied at 900 and $1800 \mathrm{MHz}$. Several different feed and short circuit locations were investigated. The simulated impedance bandwidths ( $L_{\text {retn }} \geq 6 \mathrm{~dB}$ ) are shown in Fig. 8. The significance of the chassis length is obvious and $Q_{a}$ can be estimated as described in Section II. The results also show the typical feature that larger bandwidth (i.e., stronger coupling to the chassis wavemode) is obtained, when the short circuit of the patch or PIFA is located at the top of the phone than in the opposite case where the radiating edge is located at the top.

\section{B. SAR and Radiation Efficiency Beside Human Head}

Tables I and II summarize the results for $S A R$ and radiation efficiency beside a human head obtained with finite-difference time-domain (FDTD) simulations while comparing the performance of shorted patch on a typical mobile phone-sized ground plane of $40 \mathrm{~mm} \times 110 \mathrm{~mm}$ and the case where the patch was removed and only its feed probe was used to excite the ground plane. The approximate dimensions of the patches were 
TABLE I

COMPARISON OF Simulated (FDTD) PerformanCE OF SHORTEd PATCH, MEANDERED Monopole, AND SHORT PROBE ATTACHED to $40 \mathrm{~mm}$ $\times 110 \mathrm{~mm}$ GROUND PLANE AT $915 \mathrm{MHz}$

\begin{tabular}{l|l|l|l}
\hline $\boldsymbol{f}=915 \mathrm{MHz}$ & Shorted patch & $\begin{array}{l}\text { Meandered } \\
\text { monopole }\end{array}$ & Feed probe \\
\hline Maximum $S A R(\mathrm{~W} / \mathrm{kg})$ & 13.2 & 12.3 & 12.3 \\
\hline Position $(\mathrm{X}, \mathrm{Y}, \mathrm{Z})$ & $(130,87,94)$ & $(130,87,94)$ & $(130,87,94)$ \\
\hline Average $S A R(\mathrm{~W} / \mathrm{kg})$ & 0.09 & 0.10 & 0.09 \\
\hline Maximum 1g average $S A R(\mathrm{~W} / \mathrm{kg})$ & 7.6 & 7.5 & 7.0 \\
\hline Position $(\mathrm{X}, \mathrm{Y}, \mathrm{Z})$ & $(129,88,94)$ & $(129,87,101)$ & $(129,88,94)$ \\
\hline Maximum 10g average $S A R(\mathrm{~W} / \mathrm{kg})$ & 4.5 & 4.9 & 4.2 \\
\hline Position $(\mathrm{X}, \mathrm{Y}, \mathrm{Z})$ & $(128,87,97)$ & $(127,89,104)$ & $(128,86,96)$ \\
\hline$S_{11}(\mathrm{~dB})$ & -8.4 & -7.5 & -0.01 \\
\hline Impedance & $23.6+\mathrm{j} 11.0$ & $63.7-\mathrm{j} 49.6$ & $2.2-\mathrm{j} 628.4$ \\
\hline Radiation efficiency $(\%)$ & 29.2 & 21.7 & 29.4 \\
\hline
\end{tabular}

(width $\times$ length $\times$ thickness) $40 \times 38 \times 8 \mathrm{~mm}^{3}$ at $900 \mathrm{MHz}$ and $21 \times 21 \times 8 \mathrm{~mm}^{3}$ at $1800 \mathrm{MHz}$. A meandered monopole was also used as a performance reference. The minimum distance between ground plane of the antenna and head model was $11.9 \mathrm{~mm}$. The software (XFDTD produced by Remcom, Inc.) calculates the input power, radiated power, and power dissipated in the head regardless of the matching. The $S A R$ values have been normalized to $1 \mathrm{~W}$ of $\mathrm{CW}$ input power. Fig. 9 shows the coordinates of the phone in the FDTD mesh and positions of the patches and the meandered monopole. At $900 \mathrm{MHz}$, the estimated value of $Q_{\text {eff }}$ was about 7, which means that according to (3) the power radiated by the patch wavemode is around $9 \%$, since for the thick patch the estimated value for $Q_{a}$ is about 80 . Also, in the case of the meandered monopole the coupling to the chassis wavemode was strong at $900 \mathrm{MHz}$, which can be seen from the fact that the position of the maximum $S A R$ in Table I is at the center of the chassis. As expected from the theoretical results, the type of the antenna is not significant for the $S A R$ or efficiency at $900 \mathrm{MHz}$. This can be seen from Table I, where the $S A R$ and efficiency results for the cases with patch antenna and without it (only nonradiating short-feed probe and ground plane) and also with the monopole, are practically equal. Thus, the shielding effect of the chassis, which is often regarded as the benefit of the patch antenna mounted on the backside of the phone [4], [7], does not seem significant at $900 \mathrm{MHz}$, if the antenna element is small and the bandwidth is about $10 \%$, because the relative amplitude of the patch wavemode is low. At $1800 \mathrm{MHz}$, the significance of the antenna is higher due to clearly lower $Q_{a} \approx 20$ than at $900 \mathrm{MHz}$, which can be seen both in theory from (3), which gives $P_{a} / P_{\text {tot }} \approx 50 \%$ for the simulated patch structure with $Q_{\text {eff }} \approx 10$, and from the results of Table II. Now the "directivity" of the patch (i.e., the near field shielding effect of the chassis) decreases $S A R$ and increases efficiency compared to the chassis alone. The monopole has clearly worst $S A R$ and efficiency values at $1800 \mathrm{MHz}$ due to localized absorption close to the feedpoint, though the coupling to the chassis wavemode was fairly strong also at $1800 \mathrm{MHz}$.

\section{EXPERIMENTAL RESULTS}

The modeling approach was evaluated by prototypes. The structure of a practically nonradiating planar capacitive cou-
TABLE II

Comparison of Simulated (FDTD) Performance of Shorted Patch, MEANdered Monopole, and Short Probe AtTached to $40 \mathrm{~mm}$ $\times 110 \mathrm{~mm}$ GROUND PLANE AT $1770 \mathrm{MHz}$

\begin{tabular}{|c|c|c|c|}
\hline$f=1770 \mathrm{MHz}$ & $\begin{array}{l}\text { Shorted } \\
\text { Patch }\end{array}$ & \begin{tabular}{|l} 
Meandered \\
monopole \\
$(f=1880 \mathrm{MHz})$
\end{tabular} & Feed probe \\
\hline Maximum $S A R(\mathrm{~W} / \mathrm{kg})$ & 6.9 & $\mid 19.7$ & 9.0 \\
\hline Position $(\mathrm{X}, \mathrm{Y}, \mathrm{Z})$ & $(130,88,86)$ & $(130,91,116)$ & $(130,83,116)$ \\
\hline Average $S A R(\mathrm{~W} / \mathrm{kg})$ & 0.04 & 0.07 & 0.05 \\
\hline Maximum 1g average $S A R(\mathrm{~W} / \mathrm{kg})$ & 4.1 & 11.7 & 5.4 \\
\hline Position $(\mathrm{X}, \mathrm{Y}, \mathrm{Z})$ & $(130,88,86)$ & $(129,91,113)$ & $(129,81,116)$ \\
\hline Maximum $10 \mathrm{~g}$ average $S A R(\mathrm{~W} / \mathrm{kg})$ & 2.5 & 6.5 & 2.9 \\
\hline Position $(\mathrm{X}, \mathrm{Y}, \mathrm{Z})$ & $(128,88,87)$ & $(128,90,115)$ & $(127,77,115)$ \\
\hline$\overline{S_{11}(\mathrm{~dB})}$ & -24.8 & -19.2 & -0.04 \\
\hline Impedance & $48.6-j 4.1$ & $53.1+\mathrm{j} 12.4$ & $3.6-\mathrm{j} 310.7$ \\
\hline Radiation efficiency (\%) & 65.1 & 47.7 & 57.4 \\
\hline
\end{tabular}

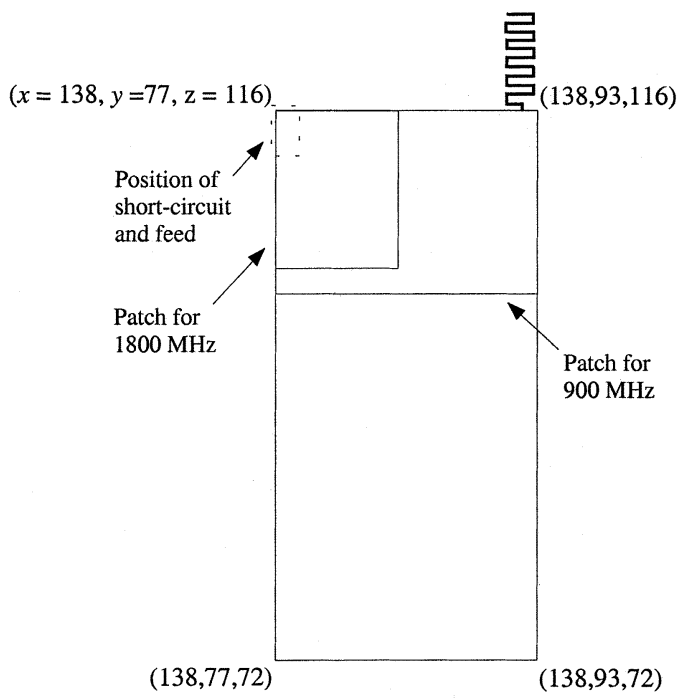

Fig. 9. Coordinates of the phone in the FDTD mesh.

pling element with microstrip resonant matching circuit was selected because it demonstrates clearly the significance of the coupling property of the "antenna" element. Microstrip technology was selected for matching circuit to minimize the volume of the coupling element by removing the inductive parts (found for example in PIFA-type elements), which do not contribute to the coupling. The circuit structure of the prototypes is shown in Fig. 10(a) and the mechanical structure of one implementation in Fig. 10(b). The reflection coefficient of the structure of Fig. 10(b) is shown in Fig. 11. The 6-dB bandwidth obtained is $5.5 \%$ giving respective $Q_{\text {eff }} \approx 24$. The obtained bandwidth is large at $980 \mathrm{MHz}$ for a planar antenna/coupling element with a volume of only $1.6 \mathrm{~cm}^{3}$. It was noticed that the bandwidth depended mainly on the volume of the coupling element, which can be expected by considering the coupling techniques of resonant cavities. Due to the $E$-field maximum in the chassis wavemode (see Fig. 1), larger bandwidth was obtained when the element was placed close to the shorter edge of the chassis. The obtained bandwidth is close to optimal, because the measured center frequency nearly equals the resonant frequency of the chassis $\left(f_{r c} \approx 1 \mathrm{GHz}\right.$ for 120 -mm-long 


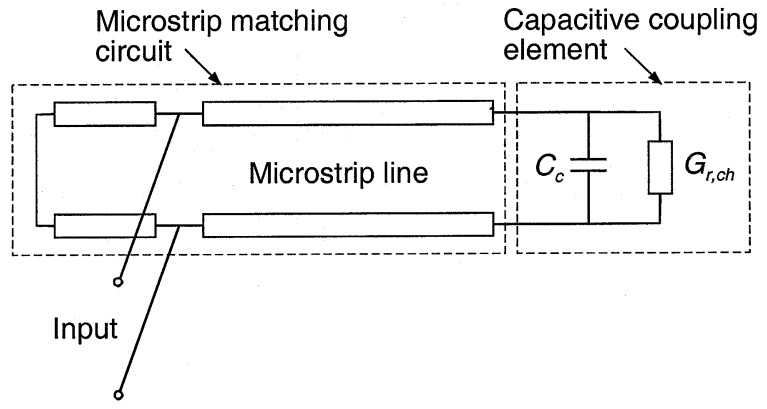

(a)

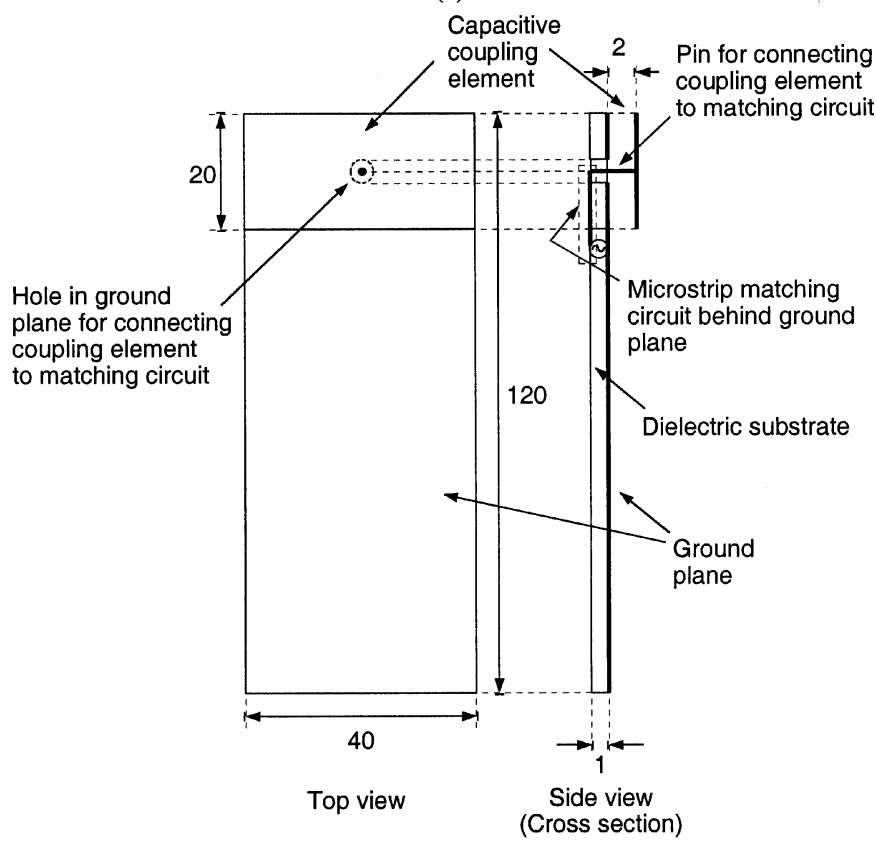

Fig. 10. (a) Circuit structure of the prototype with capacitive coupling element and microstrip matching circuit. Here $G_{\mathrm{r}, \mathrm{ch}}$ is the radiation conductance produced mainly by the chassis currents and $C_{c}$ is the capacitance of the coupling element. (b) Mechanical structure of the prototype. Thickness of the coupling element is $2 \mathrm{~mm}$ and thickness of the ground plane $1 \mathrm{~mm}$.

chassis). Based on the presented theory, the bandwidth can be expected to decrease, if the center frequency is decreased to $900 \mathrm{HMz}$, unless the chassis resonance is also decreased.

\section{DISCUSSION}

The results of this paper indicate clearly that properties like bandwidth, efficiency, $S A R$, and far-field radiation (power pattern, polarization) of the combination of antenna and chassis are defined by the amplitudes of the two wavemodes, and thus these properties are strongly connected to each other. While reporting any near- or far-field parameters of mobile-handset antennas, one should always also consider the bandwidth, which is closely connected to the relative amplitudes of the wavemodes due to their clearly different radiation quality factors. On the other hand, when reporting the bandwidth obtained with some handset antenna solution, the effect of the chassis currents should be identified. The proposed wavemode-based approach provides also possibility to draw several important further conclusions and discuss their implications for future design of mobile-handset antennas.
The impedance of the antenna-chassis combination can be described by determining the dual-resonant model of Fig. 2 separately for each frequency band of operation, if the antenna element has single resonance per frequency band. The model can be developed further by replacing the second lumped resonator representing the chassis with a distributed transmission-line model to include the combined effect of several chassis resonances. Furthermore, in the case of antenna elements having dual-resonant structure for some frequency band(s) [24], the first lumped resonator can be replaced with a dual-resonant circuit.

The impedance bandwidth of mobile handset antennas is defined especially at $900 \mathrm{MHz}$ but also at $1800 \mathrm{MHz}$ by the coupling to the chassis wavemode, which can be optimized by designing the antenna element actually to work as a coupling element. Here, well-known coupling techniques for waveguides and resonant cavities can be applied. Having chassis resonance at the frequency of operation enhances the bandwidth significantly or, optionally, makes the coupling element smaller. By maximizing the coupling one can obtain over 50\% bandwidth with optimal dual-resonant matching. For narrow impedance bandwidths, the center frequency is defined by the resonant frequency of the antenna (coupling) element but for the largest bandwidths, by the resonant frequency of the chassis.

The effect of the user on the radiation efficiency, SAR, and center frequency of the impedance bandwidth are largely defined by the interaction of the reactive near fields of the resonant wavemodes of the antenna element and the chassis with the head and hand of the user. Therefore, these phenomena can be investigated by applying resonant cavity perturbation theory [23] separately to both resonators in the system. The perturbation theory can be used to get resonance detuning and reduction of quality factor or both wavemodes. These represent the changes in impedance matching and reduction of radiation efficiency, respectively, caused by the dielectric loading of the head and hand of the user. The total effect is again obtained by determining the wavemode amplitudes, which depend on the bandwidth. At $900 \mathrm{MHz}$ and around $10 \%$ bandwidth, it is obvious that the contribution of the antenna element is so small that it does not have any significant effect on $S A R$, as seen from the results of Section IV-B. Therefore, it should be studied how the antenna elements can be replaced with clearly smaller nonradiating coupling elements like those used in the prototypes of Section V. These elements can also be placed optimally to minimize the effect of especially the hand on impedance matching. For narrowband cases $\left(B W \ll 1 / Q_{c}\right)$ detuning is caused mainly by the perturbation of the resonant frequency of the coupling element and thus, the detuning effect can be minimized by selecting the location and structure of the coupling element properly. When the contribution of the antenna element on the radiated power is significant like at $1800 \mathrm{MHz}$ and $10 \%$ bandwidth (or at $900 \mathrm{MHz}$ with $B W \ll 10 \%$ ), it is possible to reduce $S A R$ by selecting the location of the element like in the case of patch antennas on the backside of the phone. In further reduction of $S A R$ especially at $900 \mathrm{MHz}$, the chassis currents are important and controlling their distribution on the chassis should be studied. 

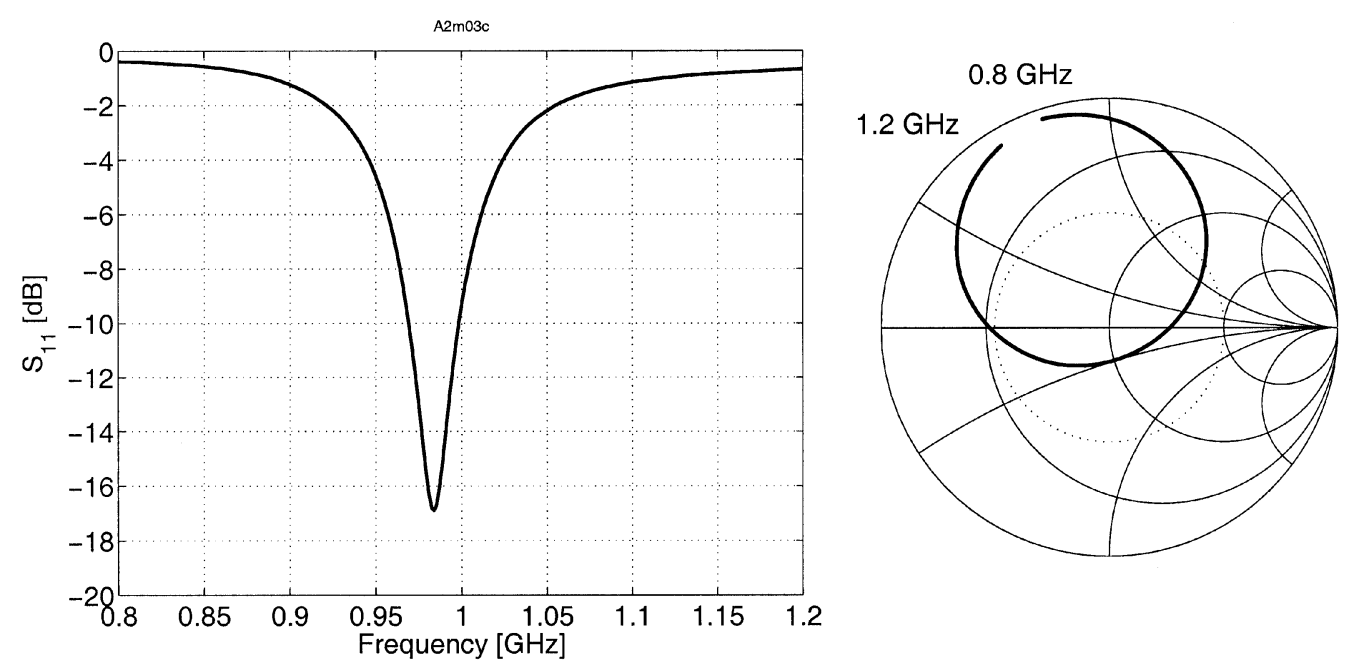

Fig. 11. Measured frequency response of reflection coefficient for the prototype of Fig. 10.

The far-field radiation properties are also defined by the contributions of the different wavemodes. Due to this the bandwidth determines, especially at $900 \mathrm{MHz}$, the possibilities to obtain such properties like directivity or polarization control, which are based on the antenna element. For example, polarization diversity at $900 \mathrm{MHz}$ can only be obtained with narrow bandwidth, because it is difficult to rotate the chassis wavemode. Increasing the size of the antenna element helps in obtaining directivity or polarization control, but at $900 \mathrm{MHz}$ this is usually impractical. Another way is to decouple the antenna element from the chassis. This leads to clearly smaller bandwidth, typically less than $1 \%$ at $900 \mathrm{MHz}$, and thus electronic tuning of the center frequency of the element is required. This increases the complexity and losses of the antenna element, but on the other hand it is possible to obtain diversity gain or higher efficiency due to shielding effect of the chassis.

\section{CONCLUSION}

In this paper, it is shown that the behavior of fairly small radio devices like mobile handsets with the maximum dimension in the range of approximately $0.25 \lambda-1 \lambda$ can be described by treating the system of antenna and chassis as a combination of the separate wavemodes of the antenna element and the chassis. An equivalent circuit was introduced for the input impedance and it was used for investigating several significant properties of the system. The results were also confirmed by electromagnetic simulations and prototyping. It was shown that by matching the resonant frequencies of the antenna element and the chassis, very large bandwidths can be obtained. It was also noticed that at $900 \mathrm{MHz}$, where the chassis wavemode typically dominates, the antenna element has only a minor effect on the properties of the near and far fields of the mobile handset. It was also shown that very small and practically nonradiating coupling elements can be used to replace the traditional self-resonant antenna elements.

\section{REFERENCES}

[1] R. J. Marhefka and W. D. Burnside, "Antennas on complex platforms," Proc. IEEE, vol. 80, pp. 204-208, Jan. 1992.
[2] N. Kuster and Q. Balzano, "Energy absorption mechanism by biological bodies in the near field of dipole antennas above $300 \mathrm{MHz}$," IEEE Trans. Veh. Technol., vol. 41, pp. 17-32, Feb. 1992.

[3] H. R. Chuang, "Human operator coupling effects on radiation characteristics of a portable communication dipole antenna," IEEE Trans. Antennas Propagat., vol. 42, pp. 556-560, Apr. 1994.

[4] M. A. Jensen and Y. Rahmat-Samii, "EM interaction of handset antennas and a human in personal communications," Proc. IEEE., vol. 83, pp. 7-17, Jan. 1995.

[5] R. Y.-S. Tay, Q. Balzano, and N. Kuster, "Dipole configurations with strongly improved radiation efficiency for hand-held transceivers," IEEE Trans. Antennas Propagat., vol. 46, pp. 798-806, June 1998.

[6] R. G. Vaughan and N. L. Scott, "Evaluation of antenna configurations for reduced power absorption in the head," IEEE Trans. Veh. Technol., vol. 48, pp. 1371-1380, Sept. 1999.

[7] G. F. Pedersen and J. B. Andersen, "Handset antennas for mobile communications: Integration diversity, and performance," in Review of Radio Science 1996-1999, W. R. Stone, Ed. New York, London, U.K.: Oxford Univ. Press, 1999, ch. 5, pp. 119-137.

[8] C. Rowell and R. Murch, "A capacitively loaded PIFA for compact mobile telephone handsets," IEEE Trans. Antennas Propagat., vol. 45, pp. 837-842, May 1997.

[9] Z. D. Liu, P. S. Hall, and D. Wake, "Dual-frequency planar inverted-F antenna," IEEE Trans. Antennas Propagat., vol. 45, pp. 1451-1458, Oct. 1997.

[10] C. Rowell and R. Murch, "A compact PIFA suitable for dual-frequency 900/1800-MHz operation," IEEE Trans. Antennas Propagat., vol. 46, pp. 596-598, Apr. 1998.

[11] J. Ollikainen, M. Fischer, and P. Vainikainen, "Thin dual-resonant stacked shorted patch antenna for mobile communications," Electron. Lett., vol. 35, no. 6, pp. 437-438, 1999.

[12] S. Nagumo, K. Kawahata, Y. Saitoh, M. Ida, and Y. Ishikawa, "Dual resonance chip dielectric antenna with a slant gap for hand held wireless communication tools," in Proc. Millennium Conf. Antennas Propagat., Davos, Switzerland, Apr. 2000, CD-ROM SP-444, European Space Agency, session 3A9.

[13] R. C. Hansen, "Fundamental limitations in antennas," Proc. IEEE, vol. 69, pp. 170-182, Feb. 1981.

[14] J. S. McLean, "A re-examination of the fundamental limits on the radiation $Q$ of electrically small antennas," IEEE Trans. Antennas Propagat., vol. 44, pp. 672-676, May 1996.

[15] T. Taga and K. Tsunekawa, "Performance analysis of a built-in planar inverted $\mathrm{F}$ antenna for $800 \mathrm{MHz}$ band portable radio units," IEEE J. Select. Areas Commun., vol. SAC-5, pp. 921-929, June 1987.

[16] K. Sato, K. Matsumoto, K. Fujimoto, and K. Hirasawa, "Characteristics of a planar inverted-F antenna on a rectangular conducting body," Electron. Commun. Japan, pt. 1, vol. 72, no. 10, pp. 43-51, 1989.

[17] T. Taga, "Analysis of planar inverted-F antennas and antenna design for portable radio equipment," in Analysis, Design and Measurement of Small and Low-Profile Antennas, K. Hirasawa and M. Haneishi, Eds. Norwood, MA: Artech House, 1992, ch. 5, pp. 161-180. 
[18] D. Manteuffel, A. Bahr, D. Heberling, and I. Wolff, "Design considerations for integrated mobile phone antennas," in Proc. 11th Int. Conf. Antennas Propagat., Apr. 17-20, 2001, pp. 252-256.

[19] P. Vainikainen, J. Ollikainen, O. Kivekäs, and I. Kelander, "Effect of mobile phone chassis on handset antenna performance," Helsinki Univ. Technol., Espoo, Finland, Radio Lab. Rep. S240 (ISBN 951-22-4928-6), Mar. 2000

[20] — , "Performance analysis of small antennas mounted on mobile handset," in Proc. COST 259 Final Workshop "The Mobile Terminal and Human Interaction", Bergen, Norway, Apr. 2000, p. 8.

[21] C. A. Balanis, Antenna Theory-Analysis and Design. New York: Wiley, 1997, p. 941.

[22] J. Ollikainen and P. Vainikainen, "Design of dual-resonant patch antennas," in Proc. 4th Eur. Personal Mobile Commun. Conf. , Vienna, Austria, Feb. 20-22, 2001, CD-ROM (ISBN 3-85 133-023-4).

[23] R. F. Harrington, Time-Harmonic Electromagnetic Fields. New York: McGraw-Hill, 1961, p. 480.

[24] J. Ollikainen, O. Kivekäs, A. Toropainen, and P. Vainikainen, "Internal dual-band patch antenna for mobile phones," in Proc. Millennium Conf. Antennas Propagat., Davos, Switzerland, Apr. 2000, CD-ROM SP-444, European Space Agency, session 3A9.

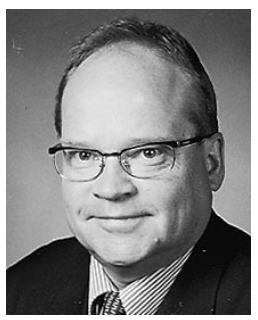

Pertti Vainikainen (M'91) was born in Helsinki, Finland, in 1957. He received the M.S., the Licentiate of Science, and the Dr. Sci. degrees in technology from Helsinki University of Technology (HUT), Espoo, Finland, in 1982, 1989, and 1991, respectively.

From 1981 to 1992 , he was with the Radio Laboratory, HUT, mainly as a Teaching Assistant and Researcher. From 1992 to 1993, he was Acting Professor of Radio Engineering, also at the Radio Laboratory, HUT. From 1993 to 1997, he was the Director of the Institute of Radio Communications (IRC), HUT. His research interests include antennas and propagation in radio communications and industrial measurement applications of radio waves. He is the author or coauthor of three books and over 110 refereed international journal or conference publications and is the holder of four patents.

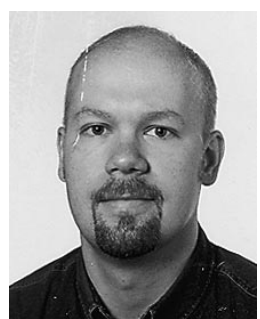

Jani Ollikainen was born in Lahti, Finland, in 1971. He received the M.S. and Licentiate of Technology degrees in electrical engineering from Helsinki University of Technology (HUT), Espoo, Finland, in 1997 and 2000, respectively. He is currently working toward the Doctorate degree at HUT.

Since 1996, he has worked as a Research Engineer at the Radio Laboratory, HUT. His research interests include design, implementation, and measurement techniques of wide-band mobile-communications antennas, especially mobile terminal antennas.

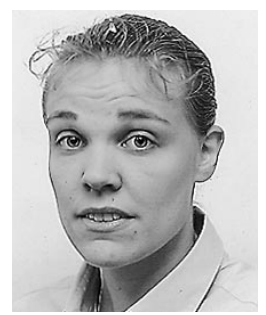

Outi Kivekäs was born in Helsinki, Finland, in 1974. She received the M.S. and Licentiate of Technology degrees in electrical engineering from Helsinki University of Technology (HUT), Espoo, Finland, in 1999 and 2001, respectively. She is currently working toward the Doctorate degree at HUT.

Since 1998, she has worked as a Research Engineer at the Radio Laboratory, HUT. Her research interests include mobile terminal antennas and their user interaction.

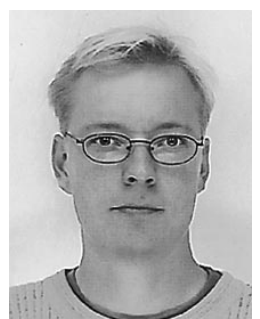

Ilkka Kelander was born in Vantaa, Finland, on March 5, 1975. He received the M.Sc. degree in electrical engineering from the Helsinki University of Technology (HUT), Espoo, Finland, in 2001.

In 2000, he joined Nokia Research Center, Helsinki, where he works on MCM-C and MCM-D packaging techniques and design of microwave components. 